\title{
Hybrid model of cardiac rehabilitation in men and women after myocardial infarction
}

\author{
Iwona Korzeniowska-Kubacka ${ }^{1}$, Maria Bilińska², \\ Barbara Dobraszkiewicz-Wasilewska ${ }^{1}$, Ryszard Piotrowicz ${ }^{1}$ \\ ${ }^{1}$ Department of Cardiac Rehabilitation and Noninvasive Electrocardiology, \\ Institute of Cardiology, Warsaw, Poland \\ ${ }^{2}$ Department of Arrhythmia, Institute of Cardiology, Warsaw, Poland
}

\begin{abstract}
Background: A relatively new alternative for post-myocardial infarction (MI) patients' care is the hybrid cardiac rehabilitation (CR) consisting of ambulatory and home-based parts. The aim of the study was to compare the influence of CR on physical capacity, safety, adherence and return to work in post-MI male and female patients with preserved left ventricular systolic function, and to assess who benefited more from this model of trainings.

Methods: The study comprised 57 men aged $54.5 \pm 7.5$ years and 30 women aged $52.2 \pm 6.7$ years after MI. All subjects underwent an 8-week training program consisting of 24 interval trainings. The first 10 trainings were conducted in an out-patient clinic, then both groups did their training at home with TeleECG monitoring. At the beginning and after trainings all patients underwent a symptom-limited exercise stress test. Assessment included results of exercise tests. Moreover, a comparative analysis of adherence and returning to work in post MI female and male patients was performed.

Results: We found that trainings led to a significant improvement in physical capacity in all patients based on exercise test. When the training effects were compared between men and women, no significant differences were observed. The percentage of patients returning to work was higher in men than in women (78.9\% vs. 50\%, $p<0.01)$.

Conclusions: 1 . Hybrid rehabilitation resulted in a comparable improvement in physical capacity in post-MI low-risk male and female patients. 2. Although hybrid rehabilitation facilitated patients' adherence to the training program, their return to work was significantly greater only in post-MI men. (Cardiol J 2015; 22, 2: 212-218)
\end{abstract}

Key words: cardiac rehabilitation, home-based tele-monitored training

\section{Introduction}

Cardiac rehabilitation (CR) is an important and integral part of the contemporary cardiac care in patients after myocardial infarction (MI). Research data show that participating in CR improves physi- cal capacity, endothelial and autonomic function, quality of life, return to work, and reduces total and cardiovascular mortality [1-6]. Despite the proven benefits, many patients after MI, especially women and older patients, are not referred to center-based CR programs.

Address for correspondence: Iwona Korzeniowska-Kubacka, MD, PhD, Department of Cardiac Rehabilitation and Noninvasive Electrocardiology, Institute of Cardiology, ul. Alpejska 42, 04-628 Warszawa, Poland, tel: +48 223434 409, fax: +48 223434 519, e-mail: drkubacka@wp.pl 
The causes why such an insufficient number of patients participate in the cardiac ambulatory rehabilitation include time-consuming commuting, difficulties in reconciling rehabilitation with professional activity, financial problems, patients' lack of motivation and physicians' insufficient involvement $[7,8]$. A chance for more patients rehabilitated is home-based monitored rehabilitation. It is important for home-based trainings to be effective and comparable with those done in a health center setting. A relatively new alternative for post-MI patients' care is the hybrid model of training, performed partly at a center and partly at home with electrocardiogram telemonitoring (TeleECG). There are only few studies assessing the efficacy and safety of this model of $\mathrm{CR}$ and their authors did not evaluate who benefited more from this kind of rehabilitation - men or women [9-11]. Moreover, women in clinical studies are still largely underrepresented. The mean percentage of women enrolled in cardiovascular clinical trials since 2006 has been $30 \%$, while only $50 \%$ of trials have reported results by gender analysis [12].

Therefore, we sought to compare the influence of hybrid CR consisting of ambulatory and home-based tele-monitored exercises on physical capacity, safety, adherence and return to work in post-MI male and female patients with preserved left ventricular systolic function, and to assess who benefited more from this model of trainings.

\section{Methods}

We analyzed 87 consecutive male and female patients aged $53.4 \pm 7.8$ years with stable angina pectoris (CCS class I or II), who were qualified for phase II of a comprehensive, post-MI, CR program.

Inclusion criteria included: age $<75$ years, sinus rhythm, preserved left ventricular function (ejection fraction $>50 \%$ ), and no evidence of right ventricular dysfunction on echocardiography, clinical stability for at least 2 weeks before the entry to the study and optimal stable medical treatment, no contraindications to stress test and patient's compliance to perform the proposed exercise program.

Patients were excluded if they had unstable angina, congestive heart failure, uncontrolled hypertension (blood pressure $>180 / 120 \mathrm{~mm} \mathrm{Hg}$ at rest), cardiac rhythm disturbances, valvular heart disease, left bundle branch block, impaired renal or hepatic function.

All patients underwent an 8-week training program consisting of 24 trainings. Before the inclusion and at the end of $\mathrm{CR}$, all patients underwent a symptom-limited exercise stress test (EST).
Moreover, a comparative analysis of returning to work in our post-MI female and male patients was assessed.

The study protocol was approved by the Institutional Ethics Committee on Human Research and each participant gave their written informed consent.

\section{Exercise stress test}

The EST was performed on a treadmill according to a modified Bruce protocol using a computerized system CASE 8000 (Marquette Electronics, Milwaukee, Wisconsin). As described previously, a 3-lead ECG was monitored continuously before, during, and for $10 \mathrm{~min}$ after the test [10]. The test was discontinued in the case of fatigue, blood pressure increase over $230 / 120 \mathrm{~mm} \mathrm{Hg}$ or a drop by more than $10 \mathrm{~mm} \mathrm{Hg}$ compared to the baseline value, ST segment depression by at least $2 \mathrm{~mm}$ and/or anginal chest pain. An EST was considered positive when ST segment depression of at least $1 \mathrm{~mm}$ was horizontal or downsloping $80 \mathrm{~ms}$ beyond the J point.

The following parameters were analyzed: maximal workload in metabolic equivalents (METs), exercise duration (ED, min), heart rate in beats per minute (HR, bpm) at rest and at peak effort, blood pressure (BP, $\mathrm{mm} \mathrm{Hg}$ ) at rest and at peak effort, double product $(\mathrm{DP}, \mathrm{mm} \mathrm{Hg} / \mathrm{min} \times 100)$, e.g., the product of $\mathrm{HR}$ and systolic $\mathrm{BP}$, at rest and at peak effort and HR recovery (HRR), i.e. the difference between $\mathrm{HR}$ at peak effort and in the $1^{\text {st }}$ minute in the recovery period. Lack of HR drop by $>12 \mathrm{bpm}$ in the $1^{\text {st }}$ minute $\left(\mathrm{HRR}_{1}\right)$ after ending EST is known to reflect diminished activity of the parasympathetic nervous system.

\section{Training program}

Patients were qualified for the program depending on the results of their initial EST.

The limit of training HR was calculated as the sum of resting HR and $60-80 \%$ of $\mathrm{HR}$ reserve, i.e. the difference between maximal HR at peak EST and $\mathrm{HR}$ at rest.

After performing the first 10 interval trainings on a cycloergometer in the ambulatory setting, patients were enrolled to a home-based telemonitored program.

\section{Interval training}

All subjects underwent an interval training on a cycloergometer 3 times a week. Each session lasted $40 \mathrm{~min}$ and included a 4-min warm-up, six 4-min exercise bouts with 2 -min rests in between, 
Table 1. Baseline characteristics of patients studied.

\begin{tabular}{lccc}
\hline & Women $(\mathbf{n}=\mathbf{3 0})$ & Men (n= 57) & $\mathbf{P}$ \\
\hline Age & $52.2 \pm 6.7$ & $54.5 \pm 7.5$ & NS \\
History of myocardial infarction & $30(100 \%)$ & $57(100 \%)$ & NS \\
CCS I & $9(31 \%)$ & $43(83 \%)$ & $<0.01$ \\
CCS II & $19(66 \%)$ & $7(14 \%)$ & $<0.01$ \\
LVEF [\%] & $60.2 \pm 8.3$ & $54 \pm 7.2$ & NS \\
Smoking & $16(56 \%)$ & $25(48 \%)$ & NS \\
Hypertension & $15(52 \%)$ & $32(62 \%)$ & NS \\
Diabetes & $3(10 \%)$ & $10(19 \%)$ & NS \\
Hypercholesterolemia & $25(86 \%)$ & $47(90 \%)$ & NS \\
Medication: & & & \\
Beta-blocker & $25(86 \%)$ & $48(92 \%)$ & NS \\
ACE-I & $25(86 \%)$ & $45(86 \%)$ & NS \\
Statins & $30(100 \%)$ & $48(92 \%)$ & NS \\
Aspirin & $30(100 \%)$ & $57(100 \%)$ & NS \\
Clopidogrel & $28(92 \%)$ & $48(92 \%)$ & NS \\
\hline
\end{tabular}

CCS - Canadian Cardiovascular Society; LVEF — left ventricular ejection fraction; ACE-I — angiotensin converting enzyme inhibitors

with gradually increased workload until the HR limit (established during the baseline EST) was reached, and a 10-min cool-down.

During each session ECG was monitored and $\mathrm{HR}$ and $\mathrm{BP}$ were measured at baseline, at the end of each interval and at recovery. The training was documented in form of a written protocol.

\section{Home-based training}

After completing the ambulatory part of trainings, patients were enrolled to home-based trainings and underwent walking training applied in an interval manner. There were three 10 -min walk exercises with 2 -min rests in between. The first 10-min period was used as a warm-up, the second was the main part of the training, and the last 10min stage was for relaxation.

All the patients were provided with $\mathrm{EHO} 3$ devices by the PRO-PLUS company. As we described previously, each patient had to place 4 electrodes on the chest following a scheme in the instruction manual, which enabled a 3-channel ECG recording to be made [10].

The devices were pre-programmed for training sessions. The pre-programming included exercise duration and intervals. When the training started, the device automatically registered resting ECG, and then, with light and sound signals, informed a patient about each training stage being started. Once each stage ended, the device recorded ECG. It was also possible to register ECG additionally during both exercise and rest. This enabled such patient's symp- toms as chest pain or palpitations to be verified. Once a training session was completed, patients transmitted the recorded ECG to the monitoring station via telephone. The monitors could assess ECG in terms of HR, arrhythmias, changes in ST segment, and verify if the training had been done properly according to the established training HR limits.

During the home-based period all patients were carefully monitored whether complications such as unstable angina, MI, heart failure and/or serious cardiac rhythm disturbances had occurred.

\section{Statistical analysis}

Statistical analysis was performed using SAS statistical software (version 8.2; Cary NC, USA). All data were expressed as mean \pm standard deviation. Student's t-test for matched pairs was used to compare the parameters of a continuous type in the two groups studied, when the distribution of variables did not differ significantly from the normal distribution; or when it did, a non-parametric rank test was used. In order to assess the differences of categorized parameters in the two groups $\chi^{2}$ test was used. A p value $<0.05$ was considered statistically significant.

\section{Results}

Baseline clinical characteristics of the patients studied are presented in Table 1.

The majority of men were in the first class according to the Canadian Cardiovascular Society 
Table 2. Exercise stress test results before and at the end of the study in men.

\begin{tabular}{lccc}
\hline & Before $(\mathbf{n}=\mathbf{5 7})$ & After $(\mathbf{n}=\mathbf{5 7})$ & P \\
\hline Maximal workload [METs] & $8.4 \pm 1.6$ & $9.9 \pm 1.9$ & $<0.001$ \\
Duration [min] & $11.5 \pm 3.4$ & $13.1 \pm 3.8$ & $<0.001$ \\
HR rest [bpm] & $70.9 \pm 10.6$ & $70.7 \pm 10.5$ & $\mathrm{NS}$ \\
HR effort [bpm] & $122.7 \pm 15.7$ & $129.3 \pm 17.7$ & $<0.001$ \\
SBP rest [mm Hg] & $113.5 \pm 14.1$ & $113.2 \pm 16.0$ & $\mathrm{NS}$ \\
DBP rest [mm Hg] & $76.6 \pm 18.0$ & $76.2 \pm 17.6$ & $\mathrm{NS}$ \\
SBP effort [mm Hg] & $166.2 \pm 26.0$ & $175.4 \pm 29.7$ & $<0.05$ \\
DBP effort [mm Hg] & $84.1 \pm 8.8$ & $86.4 \pm 9.8$ & $\mathrm{NS}$ \\
DP rest [mm Hg/min] & $81.2 \pm 16.9$ & $81.0 \pm 17.4$ & $\mathrm{NS}$ \\
DP effort [mm Hg/min] & $202.9 \pm 47.4$ & $225.2 \pm 45.7$ & $<0.05$ \\
HRR $_{1}$ & $82.4 \pm 13.8$ & $86.4 \pm 14.3$ & $\mathrm{NS}$ \\
\hline
\end{tabular}

MET — metabolic equivalent; HR — heart rate; SBP — systolic blood pressure; DBP — diastolic blood pressure; DP — double product; HRR - heart rate recovery in the $1^{\text {st }}$ minute

Table 3. Exercise stress test results before and at the end of the study in women.

\begin{tabular}{lccc}
\hline & Before $(\mathbf{n}=\mathbf{3 0})$ & After $(\mathbf{n}=\mathbf{3 0})$ & P \\
\hline Maximal workload [METs] & $7.2 \pm 2.4$ & $8.6 \pm 1.7$ & $<0.01$ \\
Duration [min] & $7.4 \pm 3.6$ & $8.6 \pm 4.0$ & $<0.01$ \\
HR rest [bpm] & $79.9 \pm 16.3$ & $79.6 \pm 13.1$ & NS \\
HR effort [bpm] & $134.0 \pm 19.2$ & $135.8 \pm 18.1$ & NS \\
SBP rest [mm Hg] & $124.3 \pm 17.2$ & $122.1 \pm 14.0$ & NS \\
DBP rest [mm Hg] & $80.3 \pm 10.3$ & $79.6 \pm 11.0$ & NS \\
SBP effort [mm Hg] & $166.2 \pm 24.4$ & $169.8 \pm 24.6$ & NS \\
DBP effort [mm Hg] & $89.7 \pm 9.3$ & $87.5 \pm 9.6$ & NS \\
DP rest [mm Hg/min] & $100.4 \pm 28.5$ & $97.0 \pm 18.2$ & NS \\
DP effort [mm Hg/min] & $224.4 \pm 50.0$ & $230.9 \pm 45.2$ & NS \\
HRR & $90.6 \pm 19.1$ & $91.3 \pm 16.9$ & NS \\
\hline
\end{tabular}

MET — metabolic equivalent; HR — heart rate; SBP — systolic blood pressure; DBP — diastolic blood pressure; DP — double product; HRR - heart rate recovery in the $1^{\text {st }}$ minute

classification of angina severity but women were predominantly in the second class. All patients had preserved left ventricular systolic function. Most enrolled patients had hypercholesterolemia and hypertension. Moreover, all patients in both groups received standard medical therapy which was maintained throughout the study.

Tables 2 and 3 demonstrate EST results before and at the end of the study in both study groups. It should be emphasized that before the study EST duration and max workload were significantly greater in men than in women suggesting their better physical capacity (duration $11.5 \pm 3.4$ vs. $7.4 \pm 3.6 \mathrm{~min}, \mathrm{p}<0.001$, and max workload $8.4 \pm 1.6$ vs. $7.2 \pm 2.4$ METs, $p<0.001$ ).

After CR was completed, maximal workload and EST duration increased significantly in both groups. Of note, at the end of the study HR, systolic $\mathrm{BP}$ and DP measured at peak exercise increased significantly only in men. The remaining EST parameters did not change significantly. Yet, when the training effects were compared between men and women based on the $\Delta \%$ increase of all the parameters analyzed, no significant differences were observed (Fig. 1).

Our study showed that patients accepted this model of trainings, including the need for interactive collaboration with the monitoring center. There were no dropouts during the study.

We did not observe any major adverse events or complications during the study.

During trainings we observed only single premature supraventricular and ventricular contractions in 13 (14.9\%) patients (10 men and 3 women). 


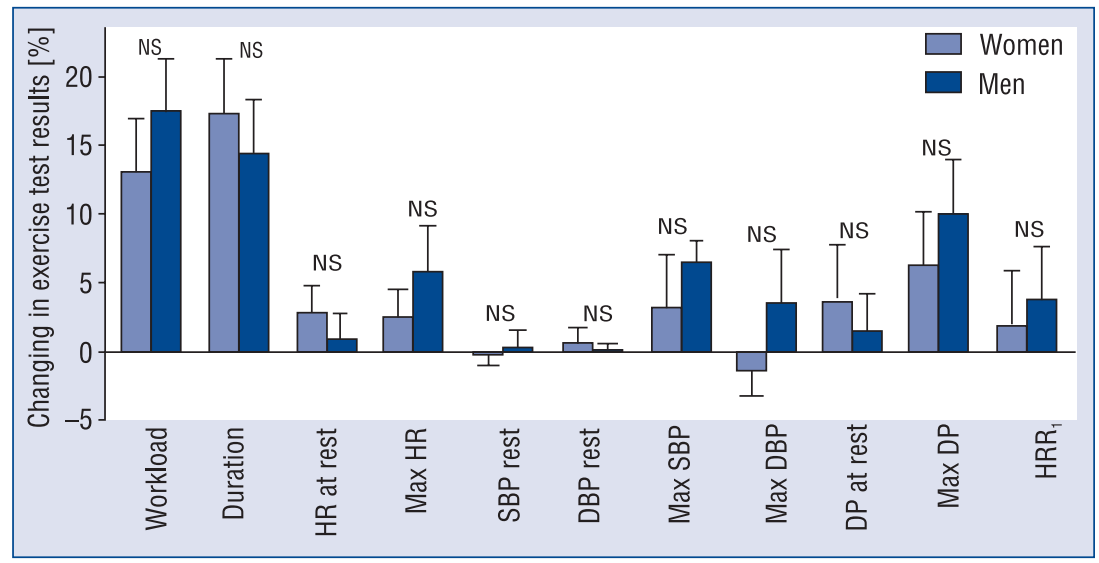

Figure 1. Changes in exercise stress test (EST) results in male and female patients who underwent a cardiac rehabilitation program. Percentage changes from the results of the baseline EST are plotted. See Table 1 for $n$ in each group; NS — non significant; HR — heart rate; DBP — diastolic blood pressure; SBP — systolic blood pressure; DP — double product, that is, product of $\mathrm{HR}$ and $\mathrm{SBP} ; \mathrm{HRR}_{1}$ - heart rate recovery in the $1^{\text {st }}$ minute after ending EST.

Table 4. The percentage of men and women who returned to work after training program.

\begin{tabular}{lccc}
\hline & Men & Women & P \\
\hline Professional work & $45(78.9 \%)$ & $15(50 \%)$ & $<0.01$ \\
Pension & $9(15.8 \%)$ & $10(33.3 \%)$ & NS \\
Mental work & $35(61.4 \%)$ & $17(56.7 \%)$ & NS \\
Physical work & $13(22.8 \%)$ & $11(36.7 \%)$ & NS \\
Inhabitants of a big city & $36(64.3 \%)$ & $20(66.7 \%)$ & NS \\
Inhabitants of a small town & $20(35.7 \%)$ & $10(33.3 \%)$ & NS \\
\hline
\end{tabular}

Our patients' return to work is presented in Table 4. We showed that a greater number of male patients than female returned to professional work after completing $\mathrm{CR}$ and their sickness benefits expired. The remaining parameters, such as the number of those receiving disability pension, type of professional work (physical vs. mental), the number of inhabitants living in a big city or small town, did not differ significantly between men and women.

Moreover, taking into account the results of final EST, we found that only men who achieved greater workload (10.2 vs. 8.6 METs, $p<0.05)$ and longer exercise duration (13.8 vs. $10.5 \mathrm{~min}$, $\mathrm{p}<0.05)$ returned to work after $\mathrm{CR}$.

\section{Discussion}

The aim of this study was to compare the effects of a hybrid model of training consisting of ambulatory and home-based tele-monitored parts in post-MI men and women and to assess who benefited more from this $\mathrm{CR}$ also in the aspect of returning to work. We found that an 8-week training program in stable coronary artery disease patients with preserved left ventricular systolic function led to a significant improvement in the physical capacity in all trained patients. When the training effects were compared between men and women, no significant differences were observed.

It is noteworthy that the home-based part of the hybrid program was monitored by a device with diode-emitted light and sound signals. This "supervision" facilitated patients to attain the training limits of HR calculated during EST. Importantly, all patients belonged to a low-risk group without left ventricular dysfunction and performed their training with intensity within $60-80 \%$ of the training HR limit. Moreover, all our patients were in touch with a physician and a physical therapist and were encouraged to train until target HR limit. Women achieved a similar increase in physical capacity as men did. It seems that the hybrid training should be dedicated to patients with a higher risk at training, e.g. heart failure patients. The low-risk post-MI patients with preserved left ventricular 
function should undergo exclusively home-based rehabilitation. Such a solution might increase the number of patients rehabilitated.

To date, there are only few articles assessing the influence of home-based CR monitored using telemedicine equipment on physical capacity in post-MI patients. Previously published studies used various modes of training during home-based $\mathrm{CR}$ and included not only coronary artery disease patients but also those with symptomatic chronic heart failure. In addition, some authors used telemonitoring mainly in order to register cardiac events, improve training safety, diagnose effortrelated ischemia and/or arrhythmia, and to modify the treatment [13].

Giallauria et al. [14] assessed the effects of exercise training in 45 patients, mainly men, after MI subdivided into 3 groups. Physical capacity improvement was comparable in patients trained in an out-patient center and at home with TeleECG monitoring, however, significantly greater than in those trained at home without telemonitoring.

Jolly et al. [15] found no differences between the center- and home-based training programs, which were monitored only by telephone contacts and nurse visits. In our papers published previously, we compared the hybrid model with the ambulatory CR separately in men and in women, and found a comparable increase in physical capacity after completing both modes of CR $[10,11]$. Despite these proven benefits of $\mathrm{CR}$, the adherence to the standard, supervised inhospital CR program remained suboptimal $[16,17]$. Therefore, to improve adherence alternative models of care such as TeleECG home-based programs have been recommended. It should be emphasized that in our study all patients accepted this model of training, including the need for collaboration with the monitoring center, and completed CR. Moreover, there were no training-related complications in either group during the study.

The results of our study are consistent with an earlier report by Scane et al. [18] describing a heterogeneous group of patients rehabilitated at home and in a center. It is noteworthy that in our study the equipment was useful not only for conducting training according to the established HR limits but also provided information about cardiac events. Moreover, home-based trainings with TeleECG monitoring facilitated the attendance of a greater number of patients at $\mathrm{CR}$ programs.

The return to work is perceived by some authors as a marker of rehabilitation efficiency [19]. The reemployment rate in our study is consistent with the previous report by Isaaz et al. [20] who showed that a greater number of men than women returned to work after $\mathrm{CR}(78 \%$ vs. $50 \%, \mathrm{p}=0.02)$. Moreover, similarly to previous study, patients who did not return to work were older and performed hard physical work before MI [19].

It should be emphasized that an improvement in physical capacity after CR was essential for returning to work in our male patients and in a study conducted by Farkas et al. [21].

In summary, the results of our study encouraged home-based CR to be applied in low-risk post-MI patients with preserved left ventricular function. These findings may have practical relevance in physical activity counselling.

\section{Limitations of the study}

Ours was a non-randomized study, which focused primarily on the comparison of the effectiveness of hybrid models of exercise therapy: home- and center-based in consecutive male and female patients after MI. Furthermore, it was only an exercise study without any assessment of cardiovascular risk factors.

Despite the study limitations listed above, our study presents a new technology which allows us to increase the low rates of patients participating in CR programs and extend their benefits to higher risk patients.

\section{Conclusions}

1. Hybrid rehabilitation resulted in a comparable improvement in physical capacity in post-MI low-risk male and female patients.

2. Although hybrid rehabilitation facilitated patients' adherence to $\mathrm{CR}$, the return to work was significantly greater only in post-MI men.

\section{Conflict of interest: None declared}

\section{References}

1. Piepoli MF, Corra U, Benzer E et al. Secondary prevention through cardiac rehabilitation: from knowledge to implementation. A position paper from the Cardiac Rehabilitation Section of the European Association of Cardiovascular Prevention and Rehabilitation. Eur J Cardiovasc Prev Rehabil, 2010; 17: 1-17.

2. Krakowska A, Kubica J, Koziński M et al. The course of early in-hospital rehabilitation in patients with acute myocardial infarction treated with primary angioplasty or thrombolytic therapy. Folia Cardiol, 2005; 4: 273-283.

3. Korzeniowska-Kubacka I, Piotrowicz R. Influence of exercise training on physical capacity, lipid profile and return to work of women after myocardial infarction. Folia Cardiol, 2004; 10: $719-725$. 
4. Cieślik A, Szykowska-Styczyrz J. The impact of early cardiological rehabilitation on life quality self-evaluation in patients after acute coronary syndrome. Kardiol Pol, 2009; 67: 583-586.

5. Flechter GF, Balady GJ, Amsterdam EA et al. Exercise standards for testing and training: A statement for healthcare professionals from the American Heart Association. Circulation, 2013; 128: 873-934.

6. Piotrowicz R, Wolszakiewicz J. Cardiac rehabilitation following myocardial infarction. Cardiol J, 2008; 5: 481-487.

7. Soleimani A, Abbasi A, Nejatian M et al. Factors predicting discontinuation of a hospital-based cardiac rehabilitation programme. Kardiol Pol, 2009; 67: 140-146.

8. Deskur-Śmielecka E, Borowicz-Bieńkowska S, Brychcy A, Wilk M, Przywarska I, Dylewicz P. Why patients after acute coronary syndromes do not participate in early outpatient rehabilitation programme? Kardiol Pol, 2009; 67: 632-638.

9. Piotrowicz E, Baranowski R, Bilińska M et al. A new model of home-based telemonitored cardiac rehabilitation in patients with heart failure: Effectiveness, quality of life and adherence. Eur J Heart Fail, 2010; 12: 164-171.

10. Korzeniowska-Kubacka I, Dobraszkiewicz-Wasilewska B, Bilińska M, Piotrowicz R. Two models of early cardiac rehabilitation in male patients after myocardial infarction with preserved left ventricular function: Comparison of standard out-patient versus hybrid training programmes. Kardiol Pol, 2011; 3: 220-226.

11. Korzeniowska-Kubacka I, Bilińska M, Dobraszkiewicz-Wasilewska B, Piotrowicz R. Comparison of home-based tele-monitored and center-based cardiac rehabilitation in female patients after myocardial infarction. A pilot study. Kardiol Pol, 2014; 72: 269-274.

12. Maas A, van der Schouw YT, Regitz-Zagrosek V et al. Red alert for women's heart: The urgent need for more research and knowledge on cardiovascular disease in women. Eur Heart J, 2011; 32: 1362-1368.

13. Scalvini S, Zanelli E, Comini L, Tomba M, Troise G. Home-based exercise rehabilitation with telemedicine following cardiac surgery. J Telemed Telecare, 2009; 15: 297-301

14. Giallauria F, Lucci R, Pilerci F et al. Efficacy of telecardiology in improving the results of cardiac rehabilitation after acute myocardial infarction. Monaldi Arch Chest Dis, 2006; 66: 1-8.

15. Jolly K, Taylor R, Lip GY et al. The Birmingham Rehabilitation Uptake Maximisation Study (BRUM). Home-based compared with hospital-based cardiac rehabilitation in a multiethnic population: cost-effectiveness and patient adherence. Health Technol Assess, 2007; 11: 1-118.

16. Beswick AD, Rees K, West RR et al. Improving uptake and adherence in cardiac rehabilitation: Literature review. J Adv Nurs, 2005; 49: 538-555.

17. Wittmer M, Volpatti M, Piazzalonga S, Hoffmann A. Expectation, satisfaction and predictors of dropout in cardiac rehabilitation. Eur J Prev Cardiol, 2012; 19: 1082-1088.

18. Scane K, Alter D, Oh P, Brooks D. Adherence to cardiac rehabilitation home program model of care: A comparison to a wellestablished traditional on-site supervised program. Appl Physiol Nutr Metab, 2012; 37: 206-213.

19. Korzeniowska-Kubacka I. Piotrowicz R. Cardiological rehabilitation: A chance of returning to work. Med Pr, 2005; 56: 325-327.

20. Isaaz K, Coudrot M, Sabry MH et al. Return to work after acute ST-segment elevation myocardial infarction in the modern area of reperfusion by direct percutaneous coronary intervention. Arch Cardiovasc Dis, 2010; 103: 310-316.

21. Farkas J, Cerne K, Lainscak M, Keber I. Return to work after myocardial infarction: Listen to your doctor. Int J Cardiol, 2008; 130: e14-e16. 